\title{
ARM7 Based Advanced Four Quadrant Sun Tracking System on Wheels with Effective power Conservation
}

\author{
Mr. Harsha. $\mathrm{P}^{1,}$ Mr. Bhupendra singh ${ }^{2}$ \\ ${ }^{1,2}$ Department of Electronics and Communication, Amity School of Engineering and technology, \\ NOIDA, U.P, INDIA
}

\begin{abstract}
In today's high-tech environment, energy has become the impetus for socio-economic development. As a free, nonpolluting, inexhaustible, renewable energy, solar energy is ideal for generating electricity. Currently, generating electricity by solar energy is inefficient and costly. This paper is a result of prototype our main project which mainly focuses on how to improve its efficiency to develop an automatic solar tracking system which will keep the solar panels aligned with the Sun in order to maximize in harvesting solar power using ARM7 microcontroller. The system tracks the maximum intensity of light. When the intensity of light is decreasing, this system automatically moves too and fro to change its position and direction to get maximum intensity of light. LDR light detector is used to trace the coordinate of the Sun. While to rotate the appropriate position of the panel, a DC geared motor is used. And also tracking system can be moved in any position where ever there is sun rays' falling on the surface of building or ground. The relay driver is used to show how to conserve the electrical energy effectively when actually there is no need of electricity.
\end{abstract}

Keyword - four quadrant, photovoltaic cells, renewable energy, LDR sensors, ARM7 microcontroller.

\section{Introduction}

These days electrical generation is typically provided by fossil fuels such as coal, natural gas, oil and also as nuclear power, hydroelectricity. Some of today's most serious environmental problems can be linked to world electricity production based primarily on the use of non-renewable resources. Currently one third of the world population do not have access to electricity and are not connected to the national grid, one solution to this problem is renewable energy in the form of photovoltaic (PV) systems. Concepts related to the solar energy have constantly been under Heavy research and development. The Basic objective is to maximize the energy produced from photovoltaic cells, by making the overall systems more efficient.

In today's existing techniques Most solar panels are statically aligned; they have a fixed position at a certain angle towards the sky. Therefore, the time and intensity of direct sunlight falling upon the solar panel is greatly reduced [2-3]. Depending on the signal operation the solar energy systems can be classified as closedloop and open loop types. An open-loop type of panel controller uses suns orbit model to adjust its tilt angle and orientation[5-7].without using feedback from the detector. Even though it is cheaper, it doesn't correct any errors so that it could not compensate for any disturbances in the system.

Where in, closed loop systems are based on feedback control principles. In these systems the sensors detect relevant parameters induced by the sun which are then transferred to controller to manipulate the panels and results maximum energy.

The prototype has been developed using closed loop type on a charci which can take the system to the place where maximum solar energy is available to convert it into electrical energy and the converted energy stored into a battery which can be used efficiently by the help of relay driver circuit actually which monitors the electricity usage, it turns on and turn off the electric devices like electric fan, air conditioner etc according to the situation by the help of some sensors like temperature sensor, light sensors and etc..

\section{System Structure}

To develop an automated sun tracking system which makes solar panel to face the sun continually as it moves across the sky from east to west and also solar panel can tilt at north and south direction, so that most of sun light will be incident to the solar panel

The proposed four quadrant sun tracking system on wheels comprises of $9 \mathrm{v}, 440 \mathrm{~mA}$ simple solar panel .and The four LDR sensors are placed on four sides of the panels in the four direction north east west and south. This set up acts like sensor module. The LDR sensors are connected to the comparator circuit which is controlled by comparator driver IC LM324 which compares the intensities across different LDR's and gives the output accordingly to the ARM7 microcontroller. Where the decision of tilting the solar panel towards the sun can be taken. The $10 \mathrm{rpm}$ geared dc motors are used to rotate the panels . the dc motors are driven by H-bridge driver circuit connected to motors and microcontroller. The H-bridge circuit acts as driver module. Four dc geared motors are used in this project. Two motors are used to move the sun tracking system towards east or 
west direction based on the maximum intensity sensed by the LDR sensors connected to the front and back end of the vehicle. And other two dc motors are used create the four quadrant model by place one dc motor over the other . the solar panel is mounted on the dc motor.

The structure of the sun tracking system on wheels is shown in Fig. 1.

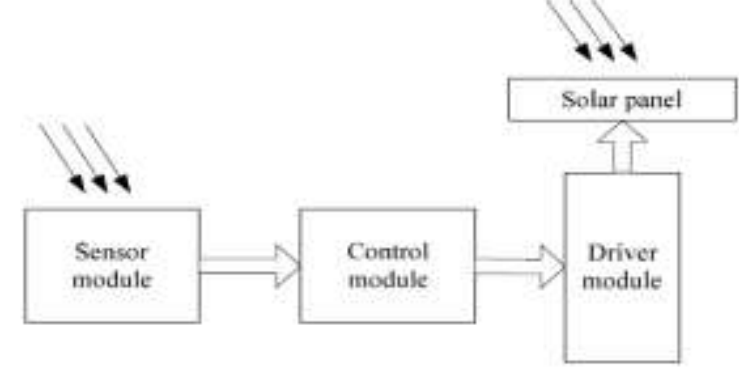

fig 1 . Sun tracking system structure

\section{A. SOLAR PANEL}

\section{Hardware Implementation}

A solar cell, sometimes called a photovoltaic cell, is a device that converts light energy into electrical energy. A single solar cell creates a very small amount of energy so solar cells are usually grouped together in an integrated electrical panel called a solar panel.

There are a number of different types of solar panels manufactured today.

1) Mono-crystalline

2) Poly-crystalline

3) Amorphous .

The solarpanel used in the project is polycrystalline $(9 \mathrm{~V} / 0.45 \mathrm{~A})$ shown in fIg. 2.

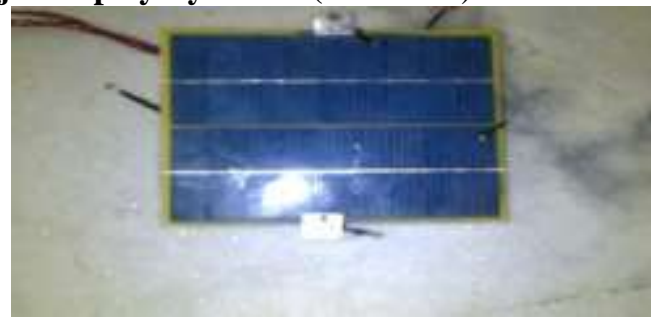

Fig.2: polycrystalline solar panel

To make polycrystalline silicon cells, liquid silicon is poured into blocks that are subsequently sawed into plates. This type of approach produces some degree of degradation of the silicon crystals which makes them less efficient. However, this type of approach is easier and cheaper to manufacture. Currently, poly-crystalline solar panels are the most common. They are slightly less efficient than single crystal, but once set into a frame with 35 or so other cells, the actual difference in $\mathrm{W} / \mathrm{m}^{2}$ is not that high. Poly-crystalline cells look somewhat like shattered glass and have a dark blue to almost black colour. Overall efficiency on average is about $11-13 \%$.

\section{B. LIGHT DETECTION SECTION}

A photo resistor or light dependent resistor is a resistor whose resistance decreases with increasing incident light intensity; in other words, it exhibits photo conductivity. It can also be referred to as a photoconductor.

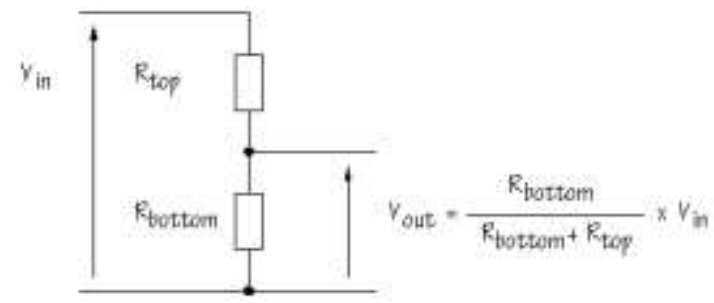

Fig 3. Voltage divider circuit

RBottom=10kohm LDR's, RTop=2.2kohm

A photo resistor is made of a high resistance semiconductor. If light falling on the device is of high enough frequency, photons absorbed by the semiconductor give bound electrons enough energy to jump into the 
conduction band. The resulting free electron (and its hole partner) conduct electricity, thereby lowering resistance.

A light sensor uses an LDR as part of a voltage divider as shown in figure 3

\section{WORKING Of LDR'S IN PROJECT}

There are 4 LDR's used in project for tracking,

- LDR1 is used to detect light in East direction. The output of voltage divider circuit varies as intensity of light falling on sensor varies, and in turn output of voltage divider circuit is given to non inverting terminal of comparator 1

- LDR2 is used to detect light in west direction. The output of voltage divider circuit varies as intensity of light falling on sensor varies, and in turn output of voltage divider circuit is given to non inverting terminal of comparator2

- LDR3 is used to detect light in south direction. The output of voltage divider circuit varies as intensity of light falling on sensor varies, and in turn output of voltage divider circuit is given to non inverting terminal of comparator3

- LDR4 is used to detect light in north direction. The output of voltage divider circuit varies as intensity of light falling on sensor varies, and in turn output of voltage divider circuit is given to non inverting terminal of comparator4.

\section{COMPARATOR}

A 'comparator' is a circuit that compares two input voltages. Reference voltages ( 2 to 3.5 volts) are given to inverting terminal and LDRs output is given to non inverting.

Working Of Comparator In This Project

LM324 is a 14 pin IC consisting of four independent operational amplifiers (op-amps) compensated in a single package. Each opamp acts as comparators in the project.

The LDR1 (East direction sensor) output is greater than reference voltage indicates light is detected in East direction.

The LDR2 (West direction sensor) output is greater than reference voltage indicates light is detected in west direction.

The LDR3 (South direction sensor) output is less than reference voltage indicates light is detected in south direction.

The LDR4 (North direction sensor) output is less than reference voltage indicates light is detected in north direction.

The LDR5 (East position sensor) output is greater than reference voltage indicates light is detected in East Position.

The LDR6 (west position sensor) output is greater than reference voltage indicates light is detected in west Position.

\section{MICROCONTROLLER}

ARM or Advanced RISC Machine, uses a 32-bit RISC (Reduced Instruction Set Computer) processor in which port p01,p02,p03,p04,p05,p08,p09 are used to connect the LDR's fixed to the solar panel and vehicle while pins p18,p19,p20,p21 of port0 are being used to connect the dc motors which helps in moving the vehicle.the pins p20,p21,p26,p27 of port 1 are used to obtain the four quadrant system which helps in keeping the solar panel perpendicular to sun's direction always.

\section{E. DC MOTOR DRIVER (L293D)}

There $4 \mathrm{dc}$ motor and 2 driver IC used in the project the output pin controller is given to DC motor driver in turn which is connected to DC motor.

- The top motor is turned on until the light is detected in X-axis (i, e East West direction) otherwise turned off.

- The base motor is turned on if there is any light detected in Y-axis(i,e north or south direction)

- $3^{\text {rd }}$ and $4^{\text {th }}$ DC motor will be turned on, if light is detected in east or west position. These motors support wheels of vehicle to move in west or east position. 


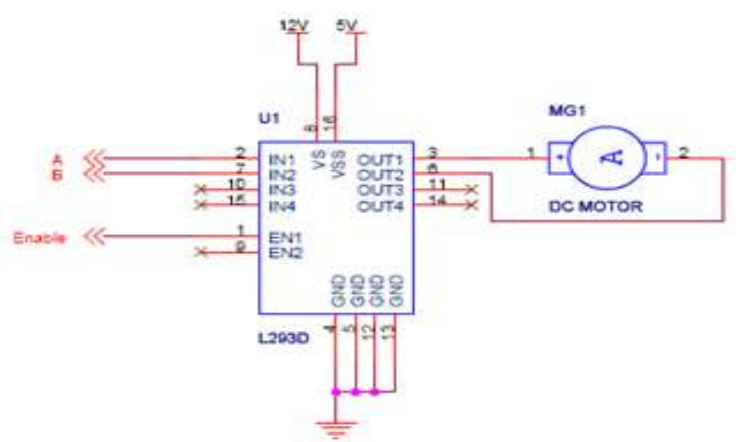

Fig.5. L293D de motor driver circuit

\section{F. RELAY DRIVER CIRCUIT}

The relay driver ULN2003E is used to show how the converted solar energy can be efficiently used in today's life when each and every unit of electricity is important to mankind. Different electrically operated home appliances can be connected to relay driver circuit where the relay input is given by different types of sensors like thermistors and ldrs . it will turn on and turn off the relays thereby the operation can be controlled accordingly. So, when there is no need for electricity the wastage can be reduced through this system.

\section{Result Of The Project}

The prototype of sun tracking system using ARM7 (lpc2148) microcontroller has been developed such that the sun tracking system being mounted on the vehicle is taken to the place where the maximum intensity is being tracked by the sensors connected to front and back end of the vehicle.

The final prototype of advanced sun tracking system is shown in fig. 6

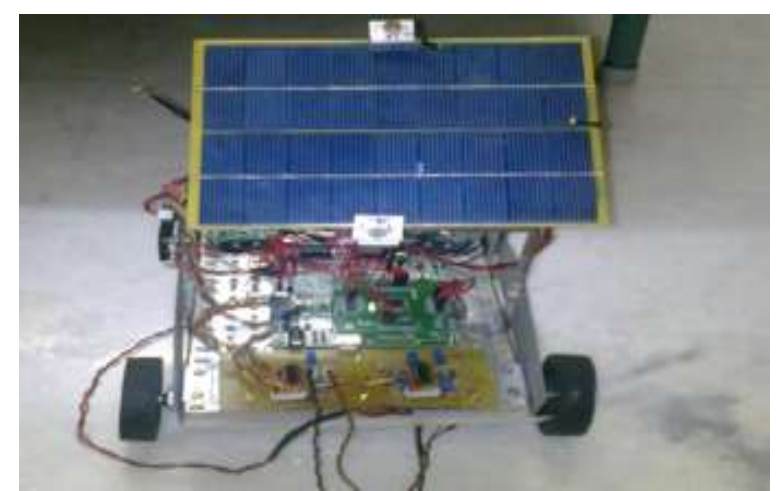

Fig 6. Prototype of "advanced sun tracking system on wheels"

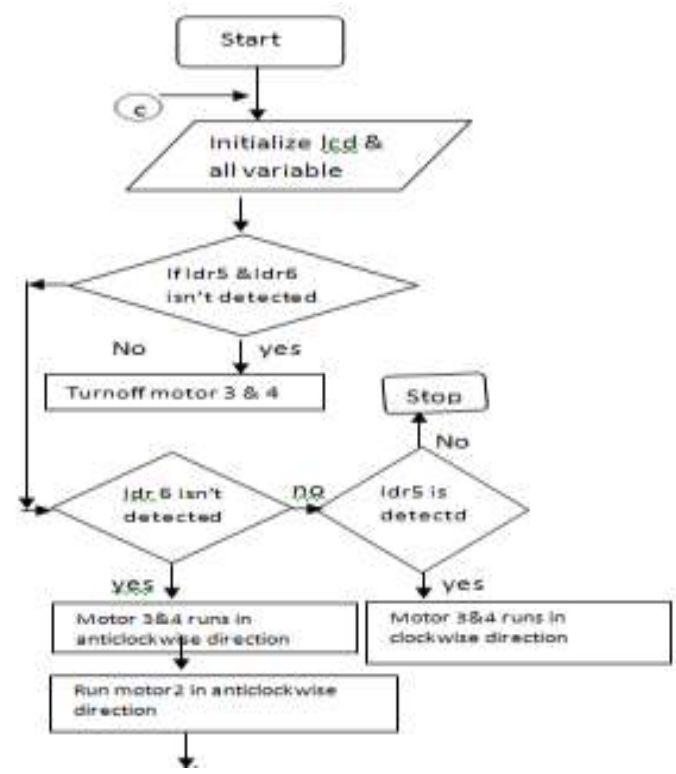



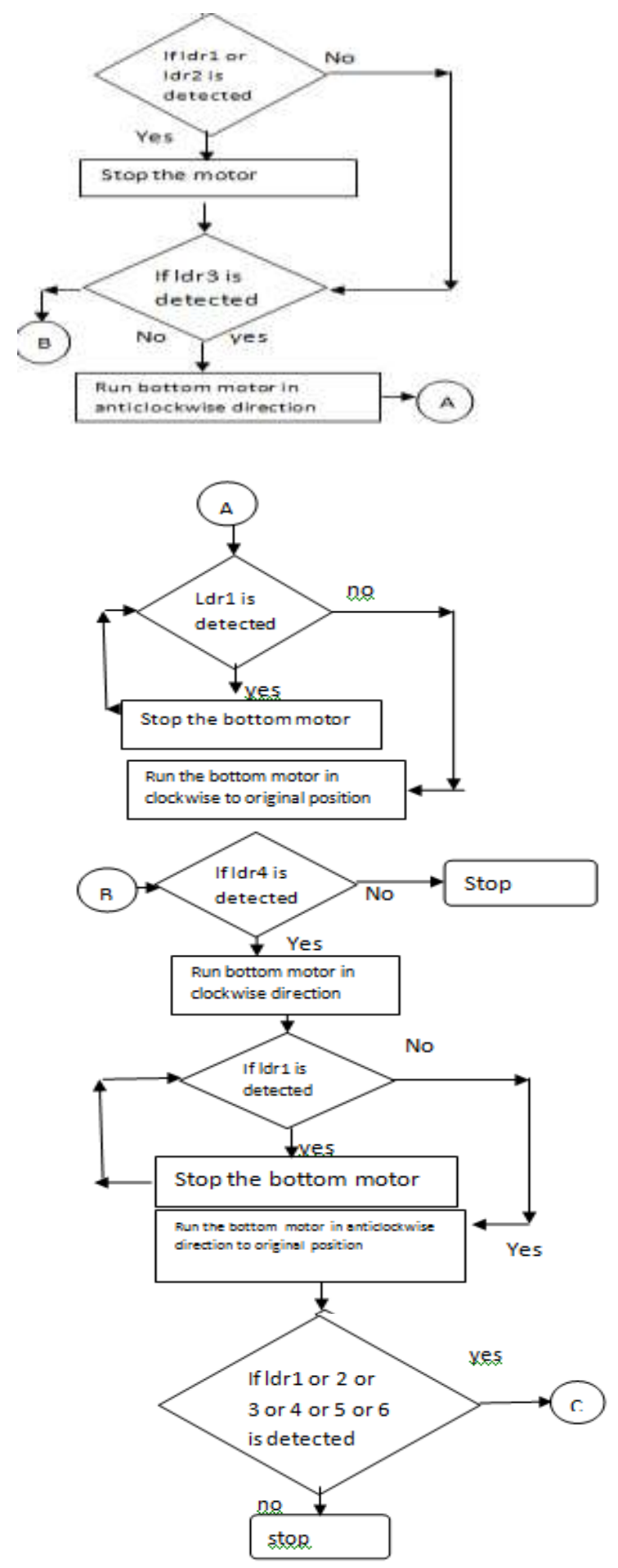

Fig 6. Flow chart of sun tracking system

\section{Conclusion \& Future Enhancement}

From the project it is evident that it is possible to improve the amount of energy that is obtained from conventional sun tracking system by $10-13 \%$ by using the advanced sun tracking system. since the proposed system is made to move to the location where the maximum intensity being sensed by the ldr sensors that are placed in front and back end of the vehicle and the solar panel made tilted to angle perpendicular to sun all the time. The relay driver circuit used in the system will show how to conserve the generated electric energy effectively for various applications.

The dc motors are directly mounted on one over the other to create the four quadrant system but in real time the mechanical gears along with dc motors are to be implemented to rotate the solar panel so that the 
weight of the solar panel will not affect the efficiency of dc motors. The only disadvantage of the system being on a vehicle is that some area has to be specially dedicated to the system to move back and forth .

\section{Refernces}

[1] Zhou Yan, Zhu Jiaxing “Application of Fuzzy Logic Control Approach in A Microcontroller-Based Sun Tracking System” 2010 WASE International Conference on Information Engineering.

[2] Chia-Yen Lee, Po-Cheng Chou, Che-Ming Chiang and Chiu-Feng Lin, "Sun Tracking Systems: A Review," Sensors, Sept. 2009, pp. 3875-3890, doi:10.3390/s90503875.

[3] Hj Mohd Yakup, M.A.; Malik, A.Q, "Optimum tilt angle and orientation for solar collector in Brunei Darussalam," Renew. Energ, vol. 24, 2001, pp. 223-234.

[4] Bari, "Optimum slope angle and orientation of solar collectors for dfferent periods of possible utilization," Energy Convers. Manage, vol. 41, 2000, pp. 855-860.

[5] McCluney, "Passive optical solar tracking system," Appl. Optics, vol. 22, 1983, pp. 3433-3439.

[6] Chen, Feng, Hong, "2006 Digital sun sensor based on the optical vernier measuring principle," Meas. Sci. Technol, vol. 17, 2006, pp. 2494-2498

[7] Chong, K.K.; Wong, C.W, "General formula for on-axis sun tracking system and its application in improving tracking accuracy of solar collector,” Sol. Energ, vol. 83, 2009, pp. 298-305.

[8] “ARM 7 MICROCONTROLLER(LPC2148)" www. Arm.com. 